\title{
ARTIGO ORIGINAL Níveis de zinco sérico em pacientes internados com depressão
}

\author{
Serum zinc levels in hospitalized patients with depression \\ Camila Saueressig', Vanuska Lima da Silva², Luciana da Conceição Antunes', Valesca Dall'Alba,
}

\section{RESUMO}

Palavras-chave

Zinco, depressão.

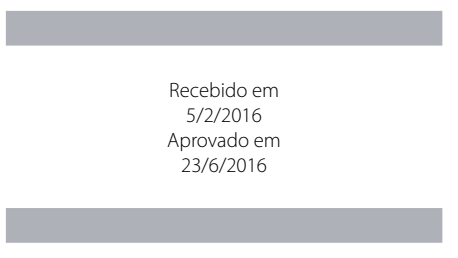

DOI: $10.1590 / 0047-2085000000130$
1 Hospital de Clínicas de Porto Alegre, Programa de Residência Integrada Multiprofissional em Saúde. 2 Universidade Federal do Rio Grande do Sul (UFRGS), Faculdade de Medicina, Departamento de Nutrição. 3 Hospital de Clínicas de Porto Alegre, Laboratório de Dor e Neuromodulação.

Endereço para correspondência: Camila Saueressig Hospital de Clínicas de Porto Alegre Rua Ramiro Barcelos, 2350 - $2^{\circ}$ andar, sala 2201 Santa Cecília - 90035-903 - Porto Alegre, RS, Brasil

E-mail: camilasaueressig@hotmail.com 


\section{Keywords}

Zinc, depression.
$81.5 \mu \mathrm{g} / \mathrm{dL}$ in THB Group, however this difference was not statistically significant. The THB Group showed higher values of IMC, LDL cholesterol and previous psychiatric hospitalizations. Conclusion: The results of this study show that serum zinc levels in patients using antidepressants and other psychiatric medications, hospitalized for bipolar disorder in depressive episode, compared with patients with depression did not differ and were within the normal range. The use of these medications may affect serum concentrations of this mineral.

\section{INTRODUÇÃO}

A depressão representa um importante e crescente problema de saúde pública. Mundialmente, estima-se que 350 milhões de pessoas sejam afetadas com a doença'. Acredita-se que seja a principal causa de incapacidade mental em termos mundiais e estima-se que, até 2020 , seja a segunda causa de incapacidade para a saúde².

A depressão pode variar em termos de gravidade, podendo ser leve, moderada ou grave, com ou sem sintomas psicóticos. Ocorre em formas episódicas, podendo se apresentar em um evento único, como também pode ser recorrente e muitas vezes crônica. Caracteriza-se por sentimento de tristeza ou irritação, anedonia, diminuição da energia, perda de confiança e autoestima, desânimo, pessimismo, sentimento de culpa, diminuição da concentração, ideias de morte e suicídio, alterações no sono e no apetite, dentre outros ${ }^{3}$. Fatores genéticos, fisiológicos, bioquímicos, morfológicos e ambientais estão associados ao desenvolvimento da doença ${ }^{4}$. Já o transtorno de humor bipolar (THB) é uma doença de curso recorrente, episódico, crônico e incapacitante, caracterizado por episódios de mania, hipomania, estados mistos e depressão ${ }^{5}$.

De maneira geral, a depressão e o THB provocam um quadro de estresse oxidativo aumentado no organismo, produzindo espécies reativas de oxigênio (ERO's), levando à oxidação de biomoléculas com perda de suas funções biológicas e/ou ao desequilíbrio homeostático ${ }^{6,7}$. O cérebro é considerado particularmente vulnerável à lesão oxidativa devido à sua elevada taxa de utilização de oxigênio e, portanto, maior geração de radicais livres ${ }^{8}$.

Em circunstâncias normais, para prevenir esses efeitos, o organismo possui mecanismos antioxidantes divididos em sistemas enzimáticos: superóxido dismutase (SOD), catalase e glutationa peroxidase, dependentes de cofatores enzimáticos como cobre, zinco e manganês; e sistema não enzimático, composto por antioxidantes dietéticos, destacando-se as vitaminas $A, C$ e $E$, minerais como cobre, zinco, manganês e selênio e compostos fenólicos ${ }^{9}$.

O zinco é um elemento-traço essencial na atividade de mais de 300 enzimas. É componente das membranas celulares e necessário para a manutenção da sua estrutura e apresenta importante função na regulação da síntese e degradação de carboidratos, proteínas, lipídeos e ácidos nucleicos, na transcrição de polinucleotídeos e consequente regu- lação da expressão gênica ${ }^{10}$. É essencial no funcionamento das estruturas cerebrais e neurais, onde a concentração mais elevada é encontrada no hipocampo e regiões da amígdala do cérebro, utilizado para modular a transmissão sináptica e também atuar como um neuromodulador endógeno de receptores importantes como o AMPA, NMDA e GABA ${ }^{11}$. Por meio de suas funções catalítica, estrutural e regulatória, desempenha importante papel na resposta imune e no estresse oxidativo ao prevenir a formação de radicais livres e proteger estruturas biológicas ${ }^{12}$.

Os dados da literatura quanto aos níveis de zinco sérico em pacientes com depressão são controversos. Enquanto alguns trabalhos demonstram uma associação inversa entre níveis de zinco e depressão ${ }^{13-16}$, em outros, os valores encontrados ficam dentro da normalidade ${ }^{17,18}$. Irmisch et al. ${ }^{18}$, apesar de não confirmarem a hipótese de níveis reduzidos de zinco sérico em transtornos depressivos, ressaltam que as concentrações do mineral diferem de acordo com a gravidade da doença.

Informações acerca do impacto da depressão e do THB sobre os níveis de zinco e no estresse oxidativo ainda são escassas. Nesse sentido, o objetivo deste trabalho foi comparar pacientes internados por depressão ou THB em episódio depressivo quanto aos níveis de zinco sérico.

\section{MÉTODOS}

Trata-se de um estudo observacional de corte transversal, realizado em uma Unidade de Internação Psiquiátrica de um hospital universitário do sul do Brasil. Foram incluídos 46 pacientes adultos, que não estivessem fazendo uso de suplemento alimentar contendo zinco. Todos os pacientes passaram por avaliação inicial da equipe por meio de detalhada anamnese e foram classificados conforme critérios diagnósticos estabelecidos pela Classificação Internacional de Doenças e Problemas Relacionados à Saúde - CID-10 ${ }^{19}$.

Para fins de comparação, os pacientes foram divididos em dois grupos: Grupo Depressão (Grupo D) e Grupo com Transtorno de Humor Bipolar em episódio depressivo (Grupo THB).

Para avaliação do zinco sérico, foram coletados $5 \mathrm{~mL}$ de sangue de cada indivíduo em um único momento, na primeira semana de internação, com mediana de 3 dias $(0-7)$, no período da tarde, sem jejum. A coleta do material foi reali- 
zada na unidade de internação por profissional do laboratório de análises clínicas do hospital, com o paciente acomodado no leito.

A amostra de sangue foi armazenada em tubos desmineralizados do tipo Trace, especiais para análise de metais. Após a coleta, o material permaneceu refrigerado por até duas horas e foi encaminhado para análise laboratorial, realizada por meio de espectrofotometria de absorção atômica. Como valores de referência para normalidade, foram adotados níveis de zinco sérico acima de $59 \mathrm{\mu g} / \mathrm{dL}$ para mulheres e acima de $61 \mu \mathrm{g} / \mathrm{dL}$ para homens ${ }^{20}$. Os demais exames bioquímicos foram acessados via prontuário eletrônico, uma vez que fazem parte da rotina da internação psiquiátrica. Entretanto, eventualmente para alguns pacientes não foram solicitados todos os exames.

A avaliação antropométrica incluiu as medidas a seguir que foram realizadas no primeiro dia de internação:

Peso: medido em quilograma $(\mathrm{kg})$ por meio da balança antropométrica eletrônica Filizola com escala de 100 gramas, com capacidade para $150 \mathrm{~kg}$. Os pacientes foram pesados descalços e com roupas leves ${ }^{21}$.

Altura: medida em metros: verificada por meio de estadiômetro fixo na parede, com o paciente descalço, de costas para o estadiômetro, com os calcanhares juntos, em posição ereta, olhando para frente e com os braços estendidos ao longo do corpo ${ }^{21}$.

O estado nutricional dos pacientes foi avaliado por meio do índice de massa corporal (IMC), definido como a relação entre o peso em quilogramas e a estatura em metros ele- vada ao quadrado $\left(\mathrm{kg} / \mathrm{m}^{2}\right)$, calculado a partir dos dados de peso e estatura. A classificação do estado nutricional foi dada de acordo com protocolos do Sistema de Vigilância Alimentar e Nutricional ${ }^{22}$.

Os participantes foram informados acerca da natureza do trabalho e assinaram o Termo de Consentimento Livre e Esclarecido. O estudo seguiu as Diretrizes e Normas Regulamentadoras de Pesquisas envolvendo Seres Humanos e foi aprovado pelo Comitê de Ética em Pesquisa do hospital onde se realizou a pesquisa, sob o número de protocolo 13-0134. Foram avaliados todos os pacientes que preencheram os critérios estabelecidos no período de abril a julho de 2015.

As variáveis categóricas são apresentadas como frequências e percentuais, e as variáveis contínuas assimétricas, como mediana e intervalo interquartil. As diferenças entre os grupos foram analisadas pelo teste não paramétrico Mann-Whitney e teste exato de Fisher para amostras independentes. Para todas as análises, o nível de significância estatística para o erro a estabelecido foi $p<0,05$. As análises foram processadas usando-se o pacote estatístico Statistical Package for the Social Sciences (SPSS) versão 20.0 (SPSS, Chicago, IL).

\section{RESULTADOS}

Na Tabela 1 estão descritas as características dos pacientes com depressão (Grupo D) e pacientes com Transtorno de Humor Bipolar em episódio depressivo (Grupo THB).

Tabela 1. Características dos pacientes com depressão (Grupo D) e Transtorno de Humor Bipolar em episódio depressivo (Grupo THB) internados em Unidade Psiquiátrica de um hospital universitário do sul do Brasil. Porto Alegre (RS), 2015

\begin{tabular}{lccc}
\hline & $D(\mathrm{n}=34)$ & THB $(\mathrm{n}=12)$ & $P$ \\
\hline Idade & $51(32-61)$ & $50(42,3-60,3)$ & 0,74 \\
Sexo masculino & $17(50 \%)$ & $7(58,3 \%)$ & 0,87 \\
IMC (kg/m) & $25,6(21,4-30,5)$ & $30,6(27,9-32,2)$ & $0,017^{*}$ \\
Tabagismo ativo & $9(26,5 \%)$ & $2(16,7 \%)$ & 0,70 \\
Uso de álcool e outras drogas (Sim) & $5(14,7 \%)$ & $1(8,3 \%)$ & 0,67 \\
Dias de permanência na unidade & $18(13,8-28,3)$ & $27,5(15-30,5)$ & 0,25 \\
Anos de doença & $2,5(0-18)$ & $6,5(0-40)$ & 0,24 \\
Internações psiquiátricas prévias (Sim) & $7(20,6 \%)$ & $11(91,7 \%)$ & $<0,01^{*}$ \\
Número de medicações psiquiátricas & $2(1-3)$ & $2,5(2-3)$ & 0,41 \\
Glicose (mg/dL)** & $88(82-94,5)$ & $91(82-113,5)$ & 0,38 \\
Colesterol total (mg/dL)** & $174,5(152,8-227,3)$ & $197(180-216,5)$ & 0,09 \\
LDL (mg/dL)*** & $100,5(89-115,4)$ & $121(104-141,5)$ & $0,04^{*}$ \\
HDL (mg/dL)*** & & & 0,84 \\
Mulheres & $41(38-60)$ & $43(39-48)$ & 0,11 \\
Homens & $43(39-45)$ & $32(27-41)$ & 0,51 \\
Triglicerídeos (mg/dL)**** & $142(99-166,5)$ & \\
\hline
\end{tabular}

D: Grupo depressão; THB: Grupo com Transtorno de Humor Bipolar em episódio depressivo. ${ }^{*}$ Nível de significância $p<0,05 .{ }^{* *} n=33$ no grupo D. ${ }^{* * *} n=22$ no grupo D. ${ }^{* * * *} n=21$ no grupo D. 
No Grupo THB, todos os pacientes apresentavam história familiar para doenças psiquiátricas e utilizavam medicações psiquiátricas anteriormente à internação. Já no Grupo D, a história familiar positiva esteve presente em praticamente 80\% dos casos e $85 \%$ já utilizavam medicações.

Em relação à gravidade da depressão, os dados estão apresentados na Tabela 2.

Tabela 2. Diagnósticos apresentados pelos pacientes com depressão (Grupo D) e Transtorno de Humor Bipolar em episódio depressivo (Grupo THB) internados em Unidade Psiquiátrica de um hospital universitário do sul do Brasil. Porto Alegre (RS), 2015

\begin{tabular}{lccc}
\hline & $\begin{array}{c}\text { D } \\
(\mathbf{n}=34)\end{array}$ & $\begin{array}{c}\text { THB } \\
(\mathbf{n}=12)\end{array}$ & $P$ \\
\hline Episódio depressivo grave sem sintomas psicóticos & $76,5 \%$ & $75 \%$ & 1,00 \\
Episódio depressivo grave com sintomas psicóticos & $11,8 \%$ & $16,7 \%$ & 0,64 \\
Episódio depressivo moderado & $2,9 \%$ & - & 1,00 \\
Episódio depressivo leve & $2,9 \%$ & - & 1,00 \\
Transtorno depressivo recorrente & $2,9 \%$ & $8,3 \%$ & 0,46 \\
Episódio depressivo não especificado & $2,9 \%$ & - & 1,00 \\
\hline
\end{tabular}

D: Grupo depressão; THB: Grupo com Transtorno de Humor Bipolar em episódio depressivo. Utilizado Teste Exato de Fisher.

Quanto ao uso de medicações, 83\% no Grupo D e 67\% no Grupo THB utilizavam antidepressivos $(p=0,45)$. A classe mais utilizada foram os inibidores seletivos da recaptação de serotonina (72\% vs. 88\%). Outras medicações utilizadas foram: antipsicóticos atípicos (71\% vs. 58\%), benzodiazepínicos (35\% vs. $42 \%$ ) e estabilizadores de humor ( $9 \%$ vs. $58 \%$ ) nos Grupos D e THB, respectivamente.

Nos pacientes avaliados, a presença de diabetes tipo 2 (DM2) foi similar nos dois grupos: 18\% no Grupo D e 17\% no Grupo THB. Um paciente de cada grupo apresentou diagnóstico de HIV.

Os níveis de zinco sérico não diferiram estatisticamente entre os grupos (Figura 1). A mediana de zinco no Grupo D foi de $88,5 \mu \mathrm{g} / \mathrm{dL}(77-103)$ e de $81,5 \mu \mathrm{g} / \mathrm{dL}(68,8-92,3)$ no Grupo THB. Os níveis de zinco sérico estavam dentro do padrão de normalidade em $95,7 \%$ dos pacientes.

Não foram encontradas associações estatisticamente significativas entre os níveis de zinco sérico e sexo, idade, IMC, uso de antidepressivos, uso de lítio, uso anterior de medicação psiquiátrica, DM2 e perfil bioquímico dos participantes do estudo (dados não apresentados).

\section{DISCUSSÃO}

A avaliação dos níveis de zinco sérico não evidenciou deficiência do mineral nos participantes do presente estudo. 0 Grupo THB apresentou valores de zinco sérico menores que o Grupo D, entretanto essa diferença não foi estatisticamente significativa.

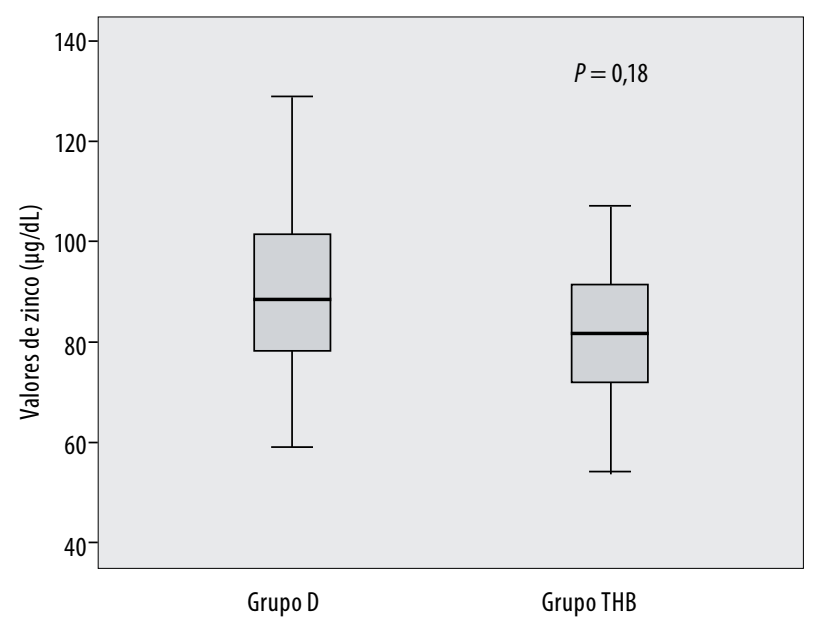

Figura 1. Correlação entre os níveis de zinco sérico entre os grupos com depressão (Grupo D) e Transtorno de Humor Bipolar em episódio depressivo (Grupo THB) internados em Unidade Psiquiátrica de um hospital universitário do sul do Brasil. Porto Alegre (RS), 2015.

Poucos estudos avaliando os níveis de zinco foram realizados no Brasil e não foram encontrados estudos em indivíduos com depressão, THB ou outras doenças psiquiátricas. Embora a análise do conteúdo de zinco sérico seja recomendada para avaliação do estado nutricional, níveis elevados ou reduzidos no soro não foram correlacionados com a concentração de zinco no cérebro, sugerindo que o total de zinco no cérebro não seria facilmente influenciado pelos níveis periféricos ${ }^{23,24}$.

No presente estudo, todos os pacientes faziam uso de antidepressivos ou outras medicações psiquiátricas, e a maioria não apresentou deficiência de zinco, sem que pudesse ser notada diferença entre os grupos. Como já demonstrado previamente, pacientes deprimidos, com deficiência de zinco, apresentaram normalização dos valores séricos após o tratamento com antidepressivos ${ }^{13,16}$.

Apesar de a literatura evidenciar deficiência de zinco em pacientes com depressão ${ }^{13-16}$, assim como no presente estudo, outros autores que avaliaram pacientes adultos internados em episódio depressivo encontraram níveis do mineral dentro do padrão de normalidade ${ }^{17,18}$.

Em parte, a deficiência de zinco em pacientes com depressão poderia ser explicada pelo sequestro de zinco por metalotioneínas (MTs) no fígado, o que parece estar relacionado a um aumento da atividade de citocinas pró-inflamatórias, como interleucina-1 e 6 (IL-1, IL-6) $)^{25}$. Entretanto, a administração crônica de fluoxetina diminui a atividade elevada de IL-6 em pacientes deprimidos, podendo atenuar a atividade do eixo hipotálamo-pituitária-adrenal, que está envolvido no estresse e depressão ${ }^{26}$, reduzindo a concentração de MTs e liberando zinco de proteínas em caso de deficiência. Tal mecanismo estaria envolvido na normalização dos níveis de 
zinco sérico após a administração crônica de antidepressivos, cujo início da ação começa a partir de duas semanas de tratamento, porém são necessárias maiores investigações. Outra ação dos antidepressivos sobre os níveis de zinco estaria relacionada ao estresse oxidativo. Foi demonstrado que pacientes com depressão apresentam níveis elevados de ERO's e que o tratamento com antidepressivos parece aumentar a atividade de enzimas antioxidantes como a SOD ${ }^{27}$, contribuindo para a normalização dos níveis do mineral.

No presente estudo, o uso de lítio não apresentou relação estatisticamente significativa com os níveis de zinco sérico. Porém, é demonstrado na literatura que, além dos antidepressivos, os estabilizadores de humor também podem exercer efeito sobre os níveis de zinco. Sussulini et al.28, ao avaliarem os níveis de zinco sérico de pacientes com THB, demonstraram que apenas voluntários não tratados com lítio apresentaram níveis reduzidos do mineral. Outros autores $^{29,30}$ sugerem que o lítio possui efeito de neuroproteção, uma vez que o tratamento diminuiu a peroxidação lipídica em pacientes com depressão bipolar, bem como aumentou a atividade da cadeia transportadora de elétrons e os níveis de óxido nítrico.

Siwek et al..$^{31}$ recomendam que a redução da concentração de zinco sérico durante um episódio depressivo agudo e a normalização dos níveis após farmacoterapia eficaz poderiam ser um marcador sensível de depressão e remissão da doença.

Quanto ao estado nutricional dos pacientes estudados, a mediana do IMC no Grupo THB evidenciou a presença de obesidade, além disso, o Grupo THB apresentou valores significativamente maiores de IMC em relação ao Grupo D. Contudo, não houve correlação estatisticamente significativa entre essa variável e os valores de zinco sérico, mesmo sabendo-se que a obesidade per se está relacionada com inflamação de baixo grau².

A gravidade dos sintomas depressivos pode variar entre os indivíduos e influenciar o consumo alimentar e os padrões de atividade física. $\mathrm{O}$ uso de medicações psiquiátricas também pode favorecer o ganho de peso, visto que alguns antipsicóticos, assim como estabilizadores de humor e alguns antidepressivos, apresentam efeitos colaterais importantes como ganho de peso, hiperglicemia, DM2 e dislipidemias ${ }^{33}$.

A obesidade está relacionada com um estado de estresse oxidativo crônico, caracterizado por um desequilíbrio entre os radicais livres formados e a presença de substâncias antioxidantes. O acúmulo de ácidos graxos no tecido adiposo parece estimular a produção de ERO's nas células adiposas por meio de NADPH oxidase, que é inibida pelo zinco ${ }^{34}$. As ERO's dentro da parede vascular contribuem para a oxidação da LDL e podem levar ao seu acúmulo nas paredes do vaso, que é citotóxico para as células endoteliais ${ }^{35}$. De fato, pacientes do Grupo THB, que eram mais obesos, também apresentaram valores superiores de LDL colesterol que o Grupo D.
Dentre as limitações deste trabalho, a avaliação do estado inflamatório por medidas mais diretas, como proteína $C$ reativa, teria sido importante para fazer referência ao aumento de estresse oxidativo, entretanto, esses dados não foram avaliados, pois não estavam disponíveis.

\section{CONCLUSÕES}

Apesar de o grupo de pacientes com THB em episódio depressivo apresentar uma mediana de zinco sérico mais baixa que o Grupo D, eles não diferiram estatisticamente. Entretanto, o Grupo THB apresentou características como número superior de internações prévias, maior IMC e níveis aumentados de colesterol $L D L$, que refletem um estado aumentado de estresse oxidativo e inflamação. $\mathrm{O}$ uso de antidepressivos e outras medicações psiquiátricas pode ter influenciado as concentrações séricas de zinco nos pacientes estudados.

Sugere-se a realização de outros estudos que avaliem os níveis de zinco nesta população, uma vez que há escassez de dados nacionais.

\section{CONTRIBUIÇÕES INDIVIDUAIS}

Camila Saueressig - Elaborou o projeto de pesquisa, coletou os dados e redigiu o artigo.

Vanuska Lima da Silva - Orientou todo o trabalho e revisou o artigo.

Valesca Dall'Alba - Orientou todo o trabalho e revisou o artigo.

Luciana da Conceição Antunes - Contribuiu significativamente na análise e interpretação dos dados.

\section{CONFLITOS DE INTERESSE}

Os autores não possuem conflitos de interesse a serem declarados.

\section{AGRADECIMENTOS}

Pelo apoio financeiro do Fundo de Incentivo à Pesquisa e Eventos (Fipe). A fonte de financiamento não estava envolvida no desenho do estudo, na coleta, análise e interpretação dos dados, na elaboração do relatório, ou na decisão de enviar o artigo para publicação.

\section{REFERÊNCIAS}

1. World Health Organization. Equity, social determinants and public health programmes [internet]. Geneva: World Health Organization, 2012 [acesso 2015 jul 14]. Disponível em: 
<http://whqlibdoc.who.int/publications/2010/9789241563970_eng.pdf>. Acesso em: 30 set. 2014

2. Murray CJL, Lopez AD (eds.). The Global Burden of Disease: A Comprehensive Assessment of Mortality and Disability from Diseases, Injuries, and Risk Factors in 1990 and Projected to 2020. Cambridge, MA: Harvard University Press; 1996.

3. American Psychiatric Association. Manual Diagnóstico e Estatístico de Transtornos Mentais: DSM-5. 5. ed. Porto Alegre: Artmed; 2014.

4. Nestler EJ, Barrot M, DiLeone RJ, Eisch AJ, Gold SJ, Monteggia LM. Neurobiology of depression. Neuron. 2002;34(1):13-25.

5. Belmaker RH. Bipolar disorder. N Engl J Med. 2004;351(5):476-86

6. Stefanescu C, Ciobica A. The relevance of oxidative stress status in first episode and recurrent depression. J Affect Disord. 2012;143(1-3):34-8.

7. Andreazza AC, Kauer-Sant'anna M, Frey BN, Bond DJ, Kapczinski F, Young LT, et al. Oxidative stress markers in bipolar disorder: a meta-analysis. J Affect Disord. 2008;111(2-3):135-44.

8. Pandya CD, Howell KR, Pillai A. Antioxidants as potential therapeutics for neuropsychiatric disorders. Prog Neuropsychopharmacol Biol Psychiatry. 2013;46:214-23.

9. Barbosa KBF, Costa NMB, Alfenas RCG, Paula SO, Minim VPR, Bressan J. Estresse oxidativo: conceito, implicações e fatores modulatórios. Rev Nutr. 2010;23(4):629-43.

10. Cozzolino SMF. Biodisponibilidade de nutrientes. 4. ed. Barueri: Manole, 2012.

11. Takeda A, Tamano H. Insight into zinc signaling from dietary zinc deficiency. Brain Res Rev. 2009;62(1):33-44

12. Stefanidou M, Maravelias C, Dona A, Spiliopoulou C. Zinc: a multipurpose trace element. Arch Toxicol. 2006;80(1):1-9.

13. Maes M, D'Haese PC, Scharpé S, D'Hondt P, Cosyns P, De Broe ME. Hypozincemia in depression. J Affect Disord. 1994;31(2):135-40.

14. Maes M, Vandoolaeghe E, Neels H, Demedts P, Wauters A, Meltzer HY, et al. Lower serum zinc in major depression is a sensitive marker of treatment resistance and of the immune/ inflammatory response in that illness. Biol Psychiatry. 1997;42(5):349-58.

15. Maes M, Christophe A, Delanghe J, Altamura C, Neels H, Meltzer HY. Lowered omega3 polyunsaturated fatty acids in serum phospholipids and cholesteryl esters of depressed patients. Psychiatry Res. 1999;85(3):275-91.

16. Nowak G, Szewczyk B. Mechanisms contributing to antidepressant zinc actions. Pol J Pharmacol. 2002;54(6):587-92.

17. Ranjbar E, Kasaei MS, Mohammad-Shirazi M, Nasrollahzadeh J, Rashidkhani B, Shams J, et al. Effects of zinc supplementation in patients with major depression: a randomized clinical trial. Iran J Psychiatry. 2013;8(2):73-9.

18. Irmisch G, Schlaefke D, Richter J. Zinc and fatty acids in depression. Neurochem Res. 2010;35(9):1376-83.

19. World Health Organization. Classificação Estatística Internacional de Doenças e Problemas Relacionados à Saúde - CID-10. Décima revisão, 2008.
20. Hotz C, Peerson JM, Brown KH. Suggested lower cutoffs of serum zinc concentrations for assessing zinc status: reanalysis of the second National Health and Nutrition Examination Survey data (1976-1980). Am J Clin Nutr. 2003;78(4):756-64.

21. Barros DC. Vigilância alimentar e nutricional - Sisvan: antropometria: como pesar e medir. 1. ed. Editora MS: Brasília, 2004.

22. Ministério da Saúde. Secretaria de Atenção à Saúde. Departamento de Atenção Básica. Orientações para coleta e análise de dados antropométricos em serviços de saúde: Norma Técnica do Sistema de Vigilância Alimentar e Nutricional - Sisvan. Brasília: Ministério da Saúde; 2011. (Série G. Estatística e Informação em Saúde).

23. Takeda A, Tamano H, Tochigi M, Oku N. Zinc homeostasis in the hippocampus of zincdeficient young adult rats. Neurochem Int. 2005;46(3):221- 5.

24. Yang Y, Jing XP, Zhang SP, Gu RX, Tang FX, Wang XL, et al. High dose zinc supplementation induces hippocampal zinc deficiency and memory impairment with inhibition of BDNF signaling. PLoS One. 2013;8(1):e55384.

25. Maes M, Mihaylova I, De Ruyter M. Lower serum zinc in Chronic Fatigue Syndrome (CFS): relationships to immune dysfunctions and relevance for the oxidative stress status in CFS. J Affect Disord. 2006;90(2-3):141-7.

26. Słuzewska A, Rybakowski JK, Laciak M, Mackiewicz A, Sobieska M, Wiktorowicz K. Interleukin-6 serum levels in depressed patients before and after treatment with fluoxetine. Ann N Y Acad Sci. 1995;762:474-6.

27. Drzyzga $Ł$, Marcinowska A, Obuchowicz E. Antiapoptotic and neurotrophic effects of antidepressants: a review of clinical and experimental studies. Brain Res Bull. 2009;79(5):248-57.

28. Sussulini A, Banzato CEM, Arruda MAZ. Exploratory analysis of the serum ionomic profile for bipolar disorder and lithium treatment. Int J Mass Spectrom. 2011;307:182-4.

29. de Sousa RT, Zarate CA, Zanetti MV, Costa AC, Talib LL, Gattaz WF, et al. Oxidative stress in early stage bipolar disorder and the association with response to lithium. J Psychiatr Res. 2014;50:36-41.

30. Shao L, Young LT, Wang JF. Chronic treatment with mood stabilizers lithium and valproate prevents excitotoxicity by inhibiting oxidative stress in rat cerebral cortical cells. Biol Psychiatry. 2005;58(11):879-84.

31. Siwek M, Dudek D, Schlegel-Zawadzka M, Morawska A, Piekoszewski W, Opoka W, et al. Serum zinc level in depressed patients during zinc supplementation of imipramine treatment. J Affect Disord. 2010;126(3):447-52.

32. Ouchi N, Parker JL, Lugus JJ, Walsh K. Adipokines in inflammation and metabolic disease. Nat Rev Immunol. 2011;11(2):85-97.

33. Toalson P, Ahmed S, Hardy T, Kabinoff G. The metabolic syndrome in patients with severe mental illnesses. Prim Care Companion. 2004;6(4):152-8.

34. Tapiero H, Tew KD. Trace elements in human physiology and pathology: zinc and metallothioneins. Biomed Pharmacother. 2003;57(9):399-411.

35. Bonetti PO, Lerman LO, Lerman A. Endothelial dysfunction: a marker of atherosclerotic risk. Arterioscler Thromb Vasc Biol. 2003;23(2):168-75. 